DOI $10.35381 / \mathrm{cm} . v 7 \mathrm{i} 1.464$

\title{
El rol de la investigación en la profesión de la Enfermería
}

\section{The role of research in the nursing profession}

Amaya Córdova Ricardo-Rosendo

brissa19831@hotmail.com

Universidad Estatal del Sur de Manabí, Jipijapa

Ecuador

https://orcid.org/0000-0002-5849-8396

Mayra Yariza Jaime-Villafuerte mayra-jv@hotmail.com

Universidad Estatal del Sur de Manabí, Jipijapa

Ecuador

https://orcid.org/0000-0003-2132-543X

Susan Stephany Chong-Manzano

susan94 chong@hotmail.com

Universidad Estatal del Sur de Manabí, Jipijapa

Ecuador

https://orcid.org/0000-0002-3267-4523

Beibelin Emily Suarez-Bastidas besb03@gamil.com

Universidad Estatal del Sur de Manabí, Jipijapa

Ecuador

https://orcid.org/0000-0002-2056-5864

Recibido: 30 de abril de 2021

Aprobado: 15 de julio de 2021 


\title{
RESUMEN
}

Objetivo: Evaluar el rol de la investigación en la profesión de la enfermería. Metodología: De corte documental - descriptiva, fundamentada en la bibliografía revisada desde el punto de vista profesional y social de la importancia de utilizar la investigación para hacer compatible la práctica de los cuidados con la evidencia científica a través de las bases de datos de Cuidatge, Cuiden, Medline, Scielo, PubMed y Cinahl. Conclusión: Se explica el rol de la investigación en la praxis de la enfermería como aquella que permite lograr fomentar la autonomía e independencia profesional y hacerla innegable en los equipos científicos interdisciplinarios para ser percibida por usuarios y la sociedad en general.

Descriptores: Investigadores; enfermería centrada en el paciente; atención primaria de salud. (Fuente: DeCS).

\begin{abstract}
ABSTRAC
Objective: To evaluate the role of research in the nursing profession. Methodology: Documentary - descriptive, based on the literature reviewed from the professional and social point of view of the importance of using research to make the practice of care compatible with scientific evidence through the databases of Cuidatge, Cuiden, Medline, Scielo, PubMed and Cinahl. Conclusion: The role of research in nursing praxis is explained as one that allows to promote professional autonomy and independence and make it undeniable in interdisciplinary scientific teams to be perceived by users and society in general.
\end{abstract}

Descriptors: Research Personnel; patient-centered care; primary health care. (Source: DeCS). 


\section{INTRODUCCION}

La investigación en el marco de la profesión de la enfermería, se considera fundamental para mejorar el conocimiento que posee el profesional de la enfermedad a través de la generación de nuevos y actualizados que inciden en las buenas prácticas de su profesión, permitiéndoles mayor autonomía en su trabajo ${ }^{1}$. Todo ello, si consideramos que durante el ejercicio de esta disciplina, son cada día mayor las exigencias de calidad del trabajo que realizan, para garantizar el cuidado y su implicación con los procesos de comprensión internos respecto de la salud y la enfermedad.

Sin embargo, cabe destacar que en los sistemas de salud se observa supremacía por la labor que desempeña el médico, debilitando la autonomía que debe regir en el personal que ejerce la enfermería para realizar trabajos de investigación que se aboquen a mejorar y dar eficacia a la práctica del mismo, sumado al poco reconocimiento que reciben por la su avanzada formación ${ }^{2}$. Por otra parte, es de indicar que tanto la gestion del cuidado como la labor asistencial que cumple este profesional, les ocupa la mayor parte del tiempo de su trabajo, alejándolo de mantenerse actualizados y ajustados a los cambios que la ciencia introduce a las teorías que deben manejar y que le son fundamentales durante la práctica de su profesión.

Cabe destacar, la posición del Consejo Internacional de Enfermeras ${ }^{3}$,quienes señalan que las asociaciones nacionales de enfermería tienen como función esencial el de promover y facilitar el proceso de investigación con los empleadores, las instituciones de formación y los organismos de financiación, mediante la colaboración, entre todos, logrando con ello el acceso a la formación a través de la indagación para impulsar el desarrollo de la ciencia y fomentar su aplicación en los cuidados del paciente. Lo expuesto, obliga a estos los profesionales descubrir distintas y mejores formas de otorgar cuidados a sus pacientes, fundamentados en el avance de los conocimientos y de los resultados obtenidos a través de la investigación en pro de garantizar un cuidado de calidad a la población que asisten convirtiéndose en un recurso humano de alta 
competencia profesional ${ }^{4}$. Con base a estas premisas, el presente artículo se centró en evaluar el rol de la investigación en la profesión de la enfermería.

\section{DESARROLLO}

Actualmente, la adquisición de conocimientos para el profesional de la enfermería, mayormente está sujeto al interés social e institucional al brindarle formación ${ }^{5}$ la cual generalmente solo es posible si se la autofinancian y realizan de forma paralela al cumplimiento de sus labores profesionales ${ }^{6}$. En este sentido, expone ${ }^{7}$ algunas barreras que se deben enfrentar para desde la investigación se puedan fortalecer los sapiencias de la enfermería, entre las que se destacan:

Barreras públicas y de contexto: la falta de recursos humanos, medios financieros y de reconocimiento social, profesional e institucional.

Barreras profesionales y personales; la falta de formación científica en los estudios básicos, escasos conocimientos para valorar y aplicar los hallazgos de la investigación., de conciencia en que la práctica habitual debe estar relacionada con las últimas investigaciones, de resistencia al cambio y hasta de celos profesionales.

Barreras comunicativas y educativas. Específicamente de incapacidad de transmitir ${ }^{8}$

Asimismo, la enfermería ha obtenido y trasferido el conocimiento considerando aspectos como:

La tradición y autoridad; donde la repetición de resultados aceptables acumula experiencia y sobre ella se asienta la tradición y el principio de autoridad del "experto". Se aceptan "verdades" institucionalizadas que están basadas en costumbres y que han sido transferidas por vía oral o escrita y con la creación de roles.

La experiencia personal e intuición; este aspecto está relacionado con la experiencia que han acumulados develado en la habilidad que demuestran para resolver los problemas que se les presentan en la práctica del trabajo de manera rápida y eficaz 


\begin{abstract}
El modelado de roles; al intentar adquirir el conocimiento modelado de las conductas de un experto.

Haciendo uso del razonamiento lógico; al hacer deducciones y explicar el conocimiento a través de hechos que consideran evidentes.
\end{abstract}

\title{
Rol de la investigación en la enfermería
}

Se indica ${ }^{9}$ que la profesión de enfermería ha experimentado una gran evolución en el marco de su rol y competencias profesionales, lo que ha hecho ineludible la disposición al cambio visible desde los estudios y basados en los hallazgos de la investigaciones desde sus prácticas profesionales, para con ello garantizar una mejor y más rápida implantación de los avances científicos en el ámbito de la gestion del cuidado. Tambien, se indica que la aplicación de la investigación en el contexto laboral de la enfermería como una herramienta para la toma de decisiones en el cuidado, permite la visibilidad de su autonomía profesional, partiendo del "querer hacer" y el "poder hacer" junto con el "disponer" de competencias investigativas que reciban desde su formación universitaria y del apoyo institucional los cuales deben proveer de los medios e infraestructura para concretar el "poder hacer" ${ }^{10}$.

Por otro lado, se resalta el hecho en la cual un profesional de la enfermería es aquel que está legalmente habilitado, responsable de sus actos profesionales, enfermera, con conocimientos y aptitudes suficientes sobre su cuerpo de doctrina y que basa su práctica en la evidencia científica ${ }^{11}$; por lo tanto, es su responsabilidad asumir la importancia que radica para su profesión el desarrollo de la investigación tomando conciencia de los beneficios que les aporta para su perfeccionamiento disciplinario y profesional. En otro orden de ideas, la investigación ${ }^{12}$ en el campo de la enfermería permite generar conocimiento científico sobre la educación en enfermería, administración, cuidados de salud, entre otros, y cuyos hallazgos inciden directa e indirectamente en sus actividades logrando aumentar, validar y perfeccionar los conocimiento de la disciplina, para 
proporcionar cuidados de calidad que deben ser constantemente evaluados y mejorados basándose en la documentación investigativa procedente de la misma

\section{METODOLOGIA}

La investigación fue de corte documental - descriptiva, fundamentada en la bibliografía revisada desde el punto de vista profesional y social de la importancia de utilizar la investigación para hacer compatible la práctica de los cuidados con la evidencia científica. Para ello, se recurrió a diferentes bases de datos o fuentes documentales, entre estos: Cuidatge, Cuiden, Medline, Scielo, PubMed y Cinahl a través de palabras claves como investigación en enfermería, barreras de la investigación y rol de la investigación en enfermería.

\section{RESULTADOS}

El desarrollo de la profesión de enfermería ha tenido un gran avance en materia de academia, pues de ser una profesión técnica a universitaria e incluso ajustada a regulaciones normativas, con autonomía, responsabilidades, cuerpo de doctrina y funciones propias, donde posee su propia facultad sanitaria, sin subordinaciones y donde se valoran y evalúan científicamente los resultados de sus práctica ${ }^{13}$ Asimismo, es una necesidad imperante la generación de sus propios cuerpos de conocimiento y para desarrollarlo hace falta avanzar en la investigación, en búsqueda de profundizar sobre el conocimiento del hombre, que les permita simplicidad en las labores de su cotidianidad profesional a fin de garantizar bienestar, confort e independencia para identificar las necesidades reales de los pacientes y dar respuesta a sus expectativas. Por otra parte, se expone ${ }^{14}$ que el convertir a la investigación en un aspecto tradicional dentro de la profesión permite fundamentar las bases de su responsabilidad profesional, para de este modo imponer en las toma de decisiones clínicas y la ejecución de actividades, información científicamente documentada. Planteamiento, que invita a incitar 
la promoción de la formación en investigación de la Enfermería para llevar a cabo estrategias y recursos que les posibiliten llevar la aplicación de sus resultados ${ }^{6}$.

\section{CONCLUSIONES}

Se aprecia que a través del rol de la investigación en la praxis de la enfermería es posible lograr fomentar la autonomía e independencia profesional y hacerla innegable en los equipos científicos interdisciplinarios para ser percibida por usuarios y la sociedad en general.

Es posible logar minimizar las barrearas que enfrentan la investigación en enfermería si se le da el mismo nivel de importancia que se le asignan a las otras áreas de la salud durante el desempeño de la práctica asistencial, para de este modo convertirlas en fortalezas en la praxis de su profesión.

El cumplimiento del rol de investigador durante la formación del profesional en enfermería les permite brindar actuar bajo un cuidado holístico, generando seguridad al paciente, liderazgo como investigador y fortalece comunicación con el equipo de trabajo.

\section{REFERENCIAS CONSULTADAS}

1. Nancy Burns; Susan K. Grove. Investigación en Enfermería. [ Nursing Research]3ª Ed. España: Elsevier; 2004.

2. Trivino Z, Sanhueza O. Paradigmas de investigación en Enfermería.

3. [Research Paradigms in Nursing.]Cienc Enferm; 11(1):17-24. [En línea] [Fecha de acceso: 5 de marzo de 2014]. URL disponible en: http://www.scielo.cl/pdf/cienf/v11n1/art03.pdf. 2005

4. International Council of Nurses. Practice professional. [Práctica Profesional] . URL disponible en: https://n9.cl/4237j 
5. Martínez JR. Barreras e instrumentos facilitadores de la enfermería basada en la evidencia. [Barriers and enabling instruments of evidence-based nursing] Enferm Clin.; 13(05): 303-308. 2003.

6. Menéndez E. El modelo hegemónico: transacciones y alternativas hacia una fundamentación teórica del modelo de auto atención en salud. [The hegemonic model: transactions and alternatives towards a theoretical foundation of the model of self-care in health] Centro de Investigaciones y Estudios Superiores en Antropología Social. México.

7. Malvárez S, Castrillón MC. Panorama de la fuerza de trabajo en Enfermería en América Latina. [Overview of the nursing workforce in Latin America.] Serie Desarrollo de Recursos Humanos. Washington, D.C.: OPS; p. 31-43.2005.

8. García-García I, Gozalbes Cravioto E. Investigación en Enfermería y en Historia de la Enfermería en España. [Research in Nursing and in History of Nursing in Spain.] Index de Enfermería. 21(1-2):100-4.

9. Silvestre Busto, C. Práctica basada en la evidencia científica: elaboración y evaluación de guías de práctica clínica y protocolos. [Evidence-based practice: development and evaluation of clinical practice guidelines and protocols] Rev. Enfermería en Anestesia, Reani-mación y Terapia del Dolor; (14): 40-44. 2006.

10. Maciá Soler, L. Investigación en Enfermería. [ Nursing Research ]Rev.Enferm. Clin ; (18) 6: 287-288. 2008.

11. Fernández $P$, Pértegas $D$. Dificultades de la Investigación en Atención Primaria. A Coruña. [Difficulties of Research in Primary Care. A Coruña]. URL disponible en: https://n9.cl//nl4

12. San Martín Rodríguez, L. Una reflexión en torno a las "ciencias de la Enfermería". [A reflection on the "nursing sciences"] Rev. Enferm. Clin. 18 (5): 262-268.2008.

13. Durán M, Editorial El Futuro de la Producción Investigativa en los Profesionales de Enfermería. [The Future of Research Production in Nursing Professionals] Rev. Cuidarte. 1(1):6-7).2010.

14. Rodríguez, F. Investigar para avanzar. [Research to advance ]Rev. Enfermería Facultativa. 147:28. 2011. 


\section{CIENCIAMATRIA}

Revista Interdisciplinaria de Humanidades, Educación, Ciencia y Tecnología

Año VII. Vol. VII. N¹. Edición Especial. 2021

Hecho el depósito de ley: pp201602FA4721

ISSN-L: 2542-3029; ISSN: 2610-802X

Universidad Nacional Experimental Francisco de Miranda (UNEFM). Santa Ana de Coro. Venezuela

Lino Rolando Rodríguez-Alegre; Rosario del Pilar López Padilla; Carlos Emiliano Gonzales-Castro

Dante Quispe-Farfán

15. Osorio X, Rivas E. Estado de la investigación en Enfermería: IX región de la Araucanía, Temuco, Chile. [State of Nursing Research: IX Araucanía Region, Temuco, Chile]. Investigación y educación en Enfermería; 23(2):82-93.2005.

(C2021 por los autores. Este artículo es de acceso abierto y distribuido según los términos y condiciones de la licencia Creative Commons Atribución-NoComercial-Compartirlgual 4.0 Internacional (CC BY-NC-SA 4.0)

(https://creativecommons.org/licenses/by-nc-sa/4.0/). 